\title{
Analysis of maternal and child health policies in Malawi: The methodological perspective
}

\section{J Daire ${ }^{1,2}$, Khalil ${ }^{2}$}

1. Faculty of Health Sciences, School of Public Health, Health Economics Unit, University of Cape Town.

2. Faculty of Nursing, University of Cape Town.

\begin{abstract}
The question of why most health policies do not achieve their intended results continues to receive a considerable attention in the literature. This is in the light of the recognized gap between policy as intent and policy as practice, which calls for substantial research work to understand the factors that improve policy implementation. Although there is substantial work that explains the reasons why policies achieve or fail to achieve their intended outcomes, there are limited case studies that illustrate how to analyze policies from the methodological perspective. In this article, we report and discuss how a mixed qualitative research method was applied for analyzing maternal and child health policies in Malawi. For the purposes of this article, we do not report research findings; instead we focus our dicussion on the methodology of the study and draw lessons for policy analysis research work. We base our disusssion on our experiences from a study in which we analyzed maternal and child health policies in Malawi over the period from 1964 to 2008. Noting the multifaceted nature of maternal and child health policies, we adopted a mixed qualitative research method, whereby a number of data collection methods were employed. This approach allowed for the capturing of different perspectives of maternal and child health policies in Malawi and for strengthening of the weaknesses of each method, especially in terms of data validity. This research suggested that the multidimensional nature of maternal and child health policies, like other health policies, calls for a combination of research designs as well as a variety of methods of data collection and analysis. In addition, we suggest that, as an emerging research field, health policy analysis will benefit more from case study designs because they provide rich experiences in the actual policy context.
\end{abstract}

\section{Introduction}

Policies are generally understood as decisions (and nondecisions) and courses of actions taken by those with responsibility in a given area, such as the health sector ${ }^{1}$. These decisions and actions affect what services are delivered and how these services are organized, financed, and implemented. Good policies do exist and are intended to improve the health and well-being of the population at large. However, there often remains a wide gap between what the policies are intended to achieve and what actually happens in practice. For example, it was noted that the progress toward the Millennium Development Goals (MDGs) is mixed and patchy, and many of the key challenges that need to be addressed relate to health ${ }^{2-4}$. For example, high rates of maternal mortality, slow improvements in rates of child survival, and a rising number of deaths from AIDS in sub-Saharan Africa continue to be cited in MDG progress reports. There are numerous factors that underlie this slow progress in achieving health-related MDGs, such as lack of investment in weak health systems, insufficient or poorly coordinated donor resources, lack of agreement on effective technical strategies, and limited scale-up of interventions that work. In addition, an area that has received less attention but contributes to slow progress in achieving the healthrelated MDGs is the analysis of how and why national health policies achieve less than expected, perform differently from expected, or even fail ${ }^{4}$.

Health policy analysis is understood as a multidisciplinary approach for understanding the factors that influence policy outcomes. It seeks to understand the processes of policy development and change in detail ${ }^{5}$. In seeking to support better policy implementation, it has been suggested that it is critical that we understand the factors that influence policy outcomes $^{6}$. Hence the value of analyzing health policies lies in the potential to provide insights as to how best to intervene in developing and implementing policy and, ultimately, influence health system strengthening and performance and public health ${ }^{6,7}$. However, policy analysis still remains an underdeveloped field of research in the health sector and has had limited application in low and middle income countries $^{5,7}$.

There has been less attention given to how to do policy analysis and what research designs, theories, or methods best inform health policy analysis ${ }^{5}$. It is argued, for instance, that despite increased recognition that policy analysis can improve policy outcomes, policy analysis is often seen as a black box ${ }^{8}$. However, recently there have been publications that are starting to provide methodological guidance as to how to do policy analysis. Most of this work still remains conceptual in that there are still few case studies of empirical work that demonstrate the methodological perspective of analyzing health policies, particularly in developing countries. In this article, therefore, we aim to report on and discuss how we applied a mixed qualitative research method approach to analyze maternal and child health $(\mathrm{MCH})$ policies in Malawi over a period of time, from 1964 to 2008. For the purposes of this paper, we do not report and discuss the study findings; instead, we focus our discussion on the methodology of the study and draw lessons for policy analysis research work, as study findings have been reported elsewhere?

\section{Methods}

The research design and approach for analyzing maternal and child health policies in Malawi. In this particular study, we analyzed the content, processes, and context for policy development and implementation, as well as the interactions between actors for MCH polices in Malawi. We focused on all government policies concerned with women's and children's health. This included, for example, national health plans, reproductive health policies, reproductive health strategies, child health policies, HIV/AIDS policy, gender, and child welfare policies. The authors selected $\mathrm{MCH}$ policies as a case study to generate insights and lessons that could be applied to other health policies in Malawi. The case study research approach involves in-depth investigation of a phenomenon in its real-life context ${ }^{10}$. This approach allowed us to generate rich information about $\mathrm{MCH}$ policies in Malawi. We looked at $\mathrm{MCH}$ policies using the elements of agenda setting, policy formulation, policy implementation, and policy evaluation as units of analysis, which made this, specifically, an embedded case study design.[10] For the reason that policy analysis seeks to understand the nature of policy, we selected a mixed methods research approach by which we purposefully combined different methods of inquiry in order to capture the different dimensions of $\mathrm{MCH}$ policies in Malawi: policy content, context, processes, and actors. We used a multistage sampling technique and a combination of various data 
collection and analysis techniques. The data, however, were predominantly qualitative. The use of qualitative data in this study was appropriate because its narrative nature had a strong potential to reveal content, context, processes, and the complexities of relationships and interactions as dimensions of MCH policies in Malawi ${ }^{11}$.

\section{Samples and sampling}

The study participants and key informants were drawn from all the three regions of Malawi in order to enhance the application of study results to all of $\mathrm{Malawi}^{12}$. The Ministry of Health $(\mathrm{MoH})$ national headquarters, public tertiary hospitals, district hospitals, and health centres were purposively selected from all the three regions as contexts from where participants and key informants were selected. In this study we used a multistage sampling technique to select the study participants and key informants. Firstly, we considered the levels and the functions of the public health system in Malawi, which includes the national headquarters, the three regional and district administrations at that time, tertiary hospitals (mainly referral hospitals), secondary hospitals (mainly district hospitals), and health centres. It was by default that we drew key informants from the $\mathrm{MoH}$ national headquarters because one of its functions is to formulate policies and oversee their implementation. Heads of departments that are involved in $\mathrm{MCH}$ policies, from formulation to implementation, were selected as key informants and interviewed.

Secondly, we selected key informants and participants from tertiary hospitals in each region (there is one tertiary hospital in each region, except in the Southern Region, where there are two tertiary hospitals. In the Southern Region, key informants and participants were selected from the oldest tertiary hospital). Hospital directors, heads of paediatric wards, heads of obstetric care, and unit matrons were selected as key informants. Ward in-changes for paediatric care, labour wards, and postnatal wards, and $\mathrm{MCH}$ coordinators were selected as study participants.

Thirdly, we selected districts from the three regions. Since we were looking for a relatively small but equal number of districts from each region, we simply divided the number of districts in each region by a denominator of three. Therefore, we selected four of the twelve districts from the Southern Region, three of the nine districts from the Central Region, and two of the six districts from the Northern Region. Then we selected specific districts using a snowball sampling technique. District health officers, district nursing officers, and hospital nursing officers were selected as key informants. Ward in-charges for labour wards, postnatal wards, and paediatric wards, as well as $\mathrm{MCH}$ coordinators, were selected as study participants.

Next, in consultation with the district health officers of the selected district hospitals, we selected two health centres from each of the selected districts, where we selected the medical assistants and one nurse from each centre as participants. Hospital directors from the Christian Health Association of Malawi (CHAM) hospitals in the selected districts were also selected as key informants. After reviewing the policy documents, we then identified key informants from nongovernmental organizations, private hospitals that are involved in $\mathrm{MCH}$ policy development and implementation, and the CHAM secretariat. A purposive sampling technique was used to select both study participants and key informants.
Participants and key informants were selected strategically so that their in-depth information would give optimal insight into lessons, gaps, and strategies for improving $\mathrm{MCH}$ in Malawi. Criteria for selecting participants were based on the level of health care facility, the profession and extent involvement in $\mathrm{MCH}$ policies of each participant, and the expected information to be collected from them through self-administered questionnaires. The criteria for selecting the key informants were primarily based on institution, followed by profession of the individual and his or her involvement in $\mathrm{MCH}$ policies, as well as expected information collected from them through key informant interviews.

\section{Data collection methods and process}

Data were collected through an official documents review, self-administered questionnaires, key informant interviews, and observations (mostly during field visits). A questionnaire, an interview guide, and a field visit guide were generated using information gathered from a comprehensive literature review, health policy analysis, and reviewing of Malawi MCH policy documents. After construction of the data collection tools and obtaining ethical approval to conduct the study, the data collection tools were piloted. Results from the pilot were analyzed and findings were utilized to refine the final questionnaire, interview guide, and field visit guide. Policy documents served as a basic source of information about $\mathrm{MCH}$ programmes, policy decisions, background or activities, and policy processes. In addition, policy documents gave the researchers ideas about important questions to pursue in the questionnaire and during interviews. Permission to access official MCH documents in Malawi was obtained from the $\mathrm{MoH}$. The various $\mathrm{MCH}$ policy documents were accessed from the MoH library, planning department, Reproductive Health Unit (RHU), Child Health Unit, and heads of departments. Official documents reviewed in this study provided information on policy content, context, process and stakeholders. The following documents were reviewed:

- National Health Plan (NHP), 1964-1969, 1973-1988, 19861995, 1999-2004

- Health Policy Framework Paper (HPFP), 1995

- A Joint Programme of Work for a Health Sector Wide Approach (SWAp) 2004 to 2010, 2004

- Programme of Child Survival and Development (CSD): Plan of Operations and Plans of Action, 1988-1992

- Reproductive Health (RH) Policy, 2002

- National RH Strategy, 2007-2011

- National Policy on Early Childhood and Development (ECD), 2001

- Road Map for Accelerating the Reduction of Maternal and Neonatal Mortality and

Morbidity in Malawi (The Road Map), 2006 and 2007 (revised version)

- IMCI Approach Policy for Accelerated Child Survival and Development (ACSD) in Malawi, 2006

- Five Year National Strategic Plan for Accelerated Child Survival and Development (ACSD) in Malawi, 2007

In addition, these other critical documents were reviewed and made reference to, to support information obtained from the above list of policy documents:

- Report of the Joint Programme Review, Maternal and Child Health, Expanded Programmes on Immunizations 
and other Elements of Primary Health Care, $1984(\mathrm{MoH}$, 1984).

- Handbook and Guide for Health Providers on the Essential Health Package (EHP) in Malawi, 2004 (MoH, 2004)

- Emergency Obstetric Care Services in Malawi: Report of a Nationwide Assessment, 2005 (MoH, 2005)

- Malawi Poverty Reduction Strategy Paper (PRSP) (Government of Malawi, 2002)

- Malawi Growth and Development Strategy (MGDS) (Government of Malawi, 2005)

- Millennium Development Goals reports, 2003,2005, 2007 (MEDP, 2003, 2005 and 2007)

- District Implementation Plans (DIPs)

- Demographic Health Surveys, 1992, 1996, 2000, 2005 (NSO, 1992, 1996, 2001 and 2005)

Use of a self-administered questionnaire allowed the researchers to gather data from a wider range of study participants. The questionnaire was completed by study participants and the data collected were used to obtain information about MCH policy processes and stakeholders' involvementin policy development. In addition, questionnaire responses provided information on respondents' experiences of $\mathrm{MCH}$ policy development and implementation. Each questionnaire was transcribed into text and sent back to respondents to verify the information. The responses from questionnaires, in addition to data from policy documents, formed a basis for issues pursued during interviews.

We conducted key informant interviews using semistructured guidelines that gave the interviews focus while also allowing interviewees to give as much information as possible within the study topic. The initial interview guide was formulated based on the reviewed literature. The final interview guide was informed by the results of the pilot study and information from documents reviewed, as well as questionnaire responses. The interviews were conducted in English and audiotaped, with permission obtained from each participant, in order to capture responses accurately. The recorded interviews were transcribed at the end of each interview session and submitted to each interviewee for verification of the information provided. Interviews were used to collect data and verify information from documents reviewed and questionnaire responses. Interview responses provided information on $\mathrm{MCH}$ policy processes and stakeholders. In addition, interview responses provided information about participants' and key informants' experiences in $\mathrm{MCH}$ policy development and implementation in Malawi. Interviews also gave us an opportunity to probe for more specific answers ${ }^{13,14}$.

Field visits were conducted to observe selected health facilities for the purposes of reality checking of $\mathrm{MCH}$ policy implementation. Information collected from field visits was intended to validate and support data gathered from the official documents and responses from the questionnaires and interviews. A number of methods were used to collect data to allow for triangulation of data form various sources. Triangulation means using more than one method to collect data on the same topic as a way of assuring the validity of research process and findings ${ }^{14,15}$. In addition, a mix of data collection methods, multistage sampling, and different types of samples were used in order to capture different dimensions of the same phenomenon ${ }^{16,17}$.

\section{Data analysis}

We used content analysis from each data collection method. This involved searching text for categories, themes, and recurring words ${ }^{18}$. This process led us through the following steps:

1. Data from the official documents review were written in a narrative form and data from questionnaires and interviews were transcribed into text statements. Phrases that pertained to $\mathrm{MCH}$ policy processes, content, context, $\mathrm{MCH}$ policy stakeholders, lessons, gaps, and strategies for addressing gaps were extracted and coded as patterns, categories, and themes, based on the study aims and objectives. Meanings were formulated from the identified and coded patterns, categories, and themes. The meanings formulated from coded themes were used as descriptions of the research findings.

2. For validity, the study descriptions were returned to the study participants and key informants (original data sources) for them to validate the findings. For example, we presented the preliminary findings at a reproductive health research dissemination meeting, organized by the $\mathrm{MoH}$, where participants critiqued and confirmed the findings.

3. A qualitative data analysis computer package, NVivo 7 , was used to capture and store data. It also facilitated coding and identification of patterns, themes and categories.[16]

4. Information obtained from the three data sources was triangulated. The process of data triangulation involved identification of common themes from the three data sources, identification of similarities and differences in the identified themes, and identification of themes only available in one data source. Additionally, triangulation of data identified divergence and convergence of information in the three data sources, and evidence of information provided in one data source without corroboration in the other two sources.

\section{Ethical considerations}

Ethical considerations applying to informed consent, the participant-researcher relationship, and gaining access to the research area and participants were observed in this study. First and foremost, ethical clearance to conduct the study in Malawi was obtained from the Health Sciences Faculty Ethics Committee of the University of Cape Town and the Health Sciences Research Council of Malawi, which is under the MoH. Following that, permission to access public health facilities was obtained from the $\mathrm{MoH}$ and individual institutions as well as the individuals participating in the study.

Individual study participants and key informants were physically approached and letters inviting them to participate in the study, by answering a self-administered questionnaire or being interviewed, were presented to them. Raw data collected for the study were only accessible to the researchers, and all scripts and questionnaires were identified by code numbers (names and official positions were not used during data collection, analysis and presentation of results). Measures to ensure confidentiality of personal information also included secured storage of data.

\section{Challenges from our experience}

Challenges encountered when carrying out the work involved to produce this paper included the difficulty http://dx.doi.org/10.4314/mmj.v27i4.4 
involved in specifying limits regarding which policies to include and discuss, because almost every heath-related policy has implications on $\mathrm{MCH}$. The researchers, therefore, had to make a deliberate effort to provide examples of $\mathrm{MCH}$ policies to be referred to at the start of each interview and in the questionnaire. In addition, the word "policy", itself, meant different things to different people. For example, key informants from international NGOs took international policies into consideration in their responses. Key informants from the $\mathrm{MoH}$ made reference to national policies, and staff from health facilities discussed policy in terms of guidelines and standard procedures for policy implementation. Although this was a challenge during data collection and analysis, it was also an advantage in the sense that the study results were informed from a broader perspective of $\mathrm{MCH}$ policies. For example, we found that $\mathrm{MCH}$ policies are influenced by international policies to a greater extent than had been expected.

In terms of data collection, the first limitation of the official documents review was that some documents were not available, including family planning policy and safe motherhood programme reports. Permission to access relevant documents for this study was sought from the $\mathrm{MoH}$ Principle Secretary. However, the $\mathrm{MoH}$ did not communicate to directors and programme coordinators that the researchers would be approaching them for relevant documents. Nevertheless, when the study was explained to responsible officials, including the fact that permission to obtain policy documents was sought from the $\mathrm{MoH}$, we were provided with the documents that were available. Another limitation was that certain data were not documented in some of the policies that were reviewed. For example, the National Health Plans for 1965-1969, 1973-1988, and 1986-1995 did not document how they were initiated. In addition, documents were highly variable in quality, with exceptional detail in some cases and virtually nothing in other programmatic components. Further to that, some documents did not provide all of the information that was required for the study because they were written for different purposes. This is why it was necessary to use other methods of data collection, using the official documents review as a base for those methods.

Although the self-administered questionnaires complemented information from the documents review, they also had their own limitations. Questionnaires were distributed and collected in person by the researchers to reduce poor return rate. This required the researchers to travel to meet the participants at their places of work. The challenge was that sometimes participants were not present at their work places for various reasons, such as being off duty or attending workshops. Attempts were made to find off-duty participants at their homes, and the researchers had to go to the certain participants' places of work on multiple occasions to catch these participants when they returned from workshops or meetings. Two questionnaires were not returned because the participants were away for three consecutive workshops. Although there was a good questionnaire return rate, 34 (3 from district hospitals and 31 from health centres) out of 88 questionnaires were not completely filled. Participants from health centres reported that they were not aware of policymaking processes. In addition, the questionnaires did not allow for probing for more information.

Key informant interviews were conducted to complement and pursue issues from the document review, interviews, and questionnaire responses. Although valuable information was collected from key informants, it is was difficult to gain access to them. This was because they were usually busy people operating under demanding time constraints. As such, appointments were rescheduled multiple times for some key informants (mainly the government officials), which was time consuming. In addition to that, some key informants specified time allocations for the interview sessions. However, the lengths of the interviews should have been determined by the issues being discussed and, since this did not always happen, the amount of data collected was often compromised. Another limitation was the likely response bias from key informants. Key informants, presumably, would want to convey an ideal situation. Some senior $\mathrm{MoH}$ staff, for example, would not want to provide answers that would reflect negatively on the performance of the ministry, with the fear of consequences if discovered. However, key informants were reassured of the confidentiality of the information they provided, and that their names and positions were not going to be included in the final report.

\section{Learning points and conclusion}

By analyzing $\mathrm{MCH}$ policies in Malawi, we learnt some lessons that can be drawn on by those interested in health policy analysis and, indeed, social public policies. Firstly, the multidimensional nature of $\mathrm{MCH}$ policies, just like any health policy, calls for a combination of research designs and methods for data collection, as well as processes for their analysis. In addition, the broader involvement of stakeholders in policy formulation and implementation necessitates selection of participants and key informants from a wide range of health system levels and institutions. Further to that, study participants and key informants should be strategically (purposively) selected to provide adequate and valid information for the research. Hence, criteria for selecting study participants and key informants can include institutional affiliation, profession, and involvement in health policies. Last but not least, a deliberate effort should be made to define the policies under study to establish a common understanding, both for the researchers and study participants.

In conclusion, we suggest that, as an emerging research field, health policy analysis will benefit more from case study designs because they provide rich experiences in the real policy context. Such rich experiences provide opportunities for generalizations beyond the specific cases investigated and, therefore, contribute to theory building.

\section{References}

1. Buse K, Mays N, Walt G. Health policy. Berkshire (UK): Open University Press; 2005.

2. United Nations. The millennium development goals report 2007. New York: United Nations; 2007.

3. United Nations. The millennium development goals report 2010. New York: United Nations; 2010.

4. Buse K, Dickinson C, Gilson L. How can the analysis of power and process in policy-making improve health outcomes? Moving the agenda forward [Internet]. London: King's College London Overseas Development Institution; 2001 [cited 200927 Aug]. Available from: www.odi.org.uk/publications/briefing/bp_oct07_health_outcomes.pdf

5. Walt G, Shiffman J, Schneider H, Murray SF, Brugha R. 'Doing' health policy analysis: methodological and conceptual reflections and challenges. Health Policy Plan. 2008 Sep;23(5):308-17. doi: 10.1093/ http://dx.doi.org/10.4314/mmj.v27i4.4 
heapol/czn024.

6. Gilson L. Implementing health system change: what are the lessons from the African Health Initiative. BMC Health Serv Res. 2013;13 Suppl 2:S14. doi: 10.1186/1472-6963-13-S2-S14.

7. Gilson L, Raphael N. The terrain of health policy analysis in low and middle income countries: a review of published literature 1994-2007. Health Policy Plan. 2008 Sep;23(5):294-307. doi: 10.1093/heapol/ czn019.

8. Hardee K, Ferreira I, Boezwinkle J, Clark B. The policy circle: a framework for analyzing the components of family planning, reproductive health, maternal health and HIV/AIDS policies. POLICY Working Paper Series No. 11. USAID; 2004.

9. Daire J, Khalil D. Health system strengthening beyond the MDGs for maternal and child health in Malawi: lessons from policy analysis. World Med Health Policy. 2010 Apr;2(1):127-94.

10. Yin R. Case study research: design and methods. Thousand Oaks (USA): SAGE Publications; 2009.

11. Bowling A. Research methods: investigating health and health services. Philadelphia: Open University Press; 1997.
12. Strydon H, Fouche CB, Delport CS. Reserach at grassroots: for the social sciences and human service professions. 3rd ed. Van Schaik: Pretoria; 2007

13. Rubin H, Rubin I. Qualitative interviewing: the art of hearing data. Thousand Oaks (USA): SAGE Publications; 2005.

14. Patton MQ. Qualitative research and evaluation methods. Thousand Oaks (USA): SAGE Publications; 2002.

15. Hansen E. Successful qualitative health research: a practical introduction. Berkshire (UK): Open University Press; 2006.

16. Kielmann K, Cataldo F, Seeley J. Introduction to qualitative research methology. United Kingdom Department for International Development (DFID); 2011.

17. Maree K. First steps in research. Pretoria: Van Schaik Publishers; 2007.

18. Strauss AL, Corbin J. Basics of qualitative research: techniques and procedures for developing grounded theory. Thousand Oaks (USA): SAGE Publications; 1998. 